\title{
Constituent quark masses from a modified perturbative QCD
}

\author{
Alejandro Cabo Montes de Oca \\ Instituto de Cibernética, Matemática y \\ Física; Calle E, No. 309, Vedado, La Habana, Cuba and \\ International Institute for Theoretical and Applied Physics, \\ UNESCO and Iowa State University, Ames, Iowa 50011, U.S.A. \\ Marcos Rigol \\ Centro de Estudios Aplicados al Desarrollo Nuclear; \\ Calle 30, N. 502 e/ 5ta y 7ma, Miramar, La Habana, Cuba
}

\begin{abstract}
A recently proposed modified perturbative expansion for QCD incorporating gluon condensation is employed to evaluate the quark and gluon self-energy corrections in the first approximations. The results predict mass values of $1 / 3$ of the nucleon mass for the light quarks $u, d$, and $s$ and a monotonously growing variation with the current mass. The only phenomenological input consists in that $\left\langle G^{2}\right\rangle$ is evaluated up to order $g^{2}$ as a function of the unique parameter $C$ defining the modified propagator and then $C$ is fixed to give a current estimate of $\left\langle g^{2} G^{2}\right\rangle$. The light quarks $u$ and $d$ result to be confined and the $s, c, b$ and $t$ ones show damped propagation modes, then suggesting a model for the large differences in stability between the nucleons and the higher resonances. The above properties of quark modes diverge from the fully confinement result following from the similar gluon propagator previously considered by Munczek and Nemirovski. On another hand, the condensate effects on the gluon self-energy furnish a tachionic mass shell as predicted by the Fukuda analysis of gluon condensation in QCD.
\end{abstract}

PACS numbers: 12.38.-t, 12.38.Bx, 12.40.Yx, 12.38.Aw, 12.15.Ff 


\section{INTRODUCTION}

One of the great achievements of theoretical High Energy Physics in the last thirty years has been the discovering and development of the Quantum Chromodynamics (QCD). The smallness of the coupling constant at high momenta (asymptotic freedom) has allowed to develop a perturbative framework applicable for very high energy processes. That situation strongly simplifies the study of such phenomena and the calculated quantities are in good agreement with the experimental data. However, perturbative QCD (PQCD) is far from being able to furnish even a rough description for the relevant physics at low energies. The solution of this situation is currently one of the main challenges of Particle Physics.

A relevant phenomenon related with QCD is the color confinement. Nowadays, there are strong reasons to believe that the relation between the basic quantities in QCD, like the gluon and quarks fields, and the real world characterized by a whole variety of interacting mesons and baryons, can be understood by solving the confinement problem. The basic picture which seems plausible to be derived from QCD is that the fundamental fields can not be associated to asymptotic states of quark and gluons and then the true physical states consist only of colorless composites of quarks and gluons (mesons and baryons).

The above quoted limitations of the PQCD mean in particular that the usual Fock space vacuum of the non-interacting theory, is unable to predict, even approximately, the real ground state properties of QCD [1, 2, 3, 4]. This is at contrast with the case in QED where the standard perturbation theory gives a better than good concordance with the experimental data. Then, the nature of vacuum structure is one of the main problems to be clarified and naturally its solution is closely linked with the color confinement effect. Reviews of various models considered to investigate the confinement and the vacuum structure in QCD can be found in Refs. [5, 6].

Another basic problem for QCD is the one related with the quark masses, that is, the so called current quarks masses which appear in the Lagrangian. As the quarks are not observed as free states, the meaning of the quark masses need to be considered with care [7, 8, 9]. The nature of these parameters is an important object of research since long time ago [10, 11, 12]. The relations among these masses and their magnitude have been determined through the methods of current algebra in combination with measurements of the light mesons masses. 
In the former works [13, 14, 15] an attempt directed to construct a modified perturbation expansion for QCD being able to predict at least some low energy properties of this theory have been considered. First, in [13], the modified expansion conserving the color $S U(3)$ and Lorentz symmetries was proposed, aiming to solve the symmetry limitations of the earlier chromo-magnetic field models [1, 2, 3, 团] which inspired the search. A similar lack of manifest Lorentz invariance had also a modification of the Feynman rules, intending to include gluon condensation, advanced later in [16, 17, 18] in which a delta function for $k<\Lambda_{q c d}$ was summed to the perturbative piece. The expansion proposed in [13], considers a change of the gluon propagator in a term associated to a condensation of zero momentum gluons. This alteration of the usual rules had the interesting property of producing a non vanishing value for the gluon condensation parameter $\left\langle g^{2} G^{2}\right\rangle$ already in the first approximations [19]. In addition, in [13] a non vanishing value for the effective self-energy of the gluons was obtained at the loop level. Finally, a perturbative evaluation of the effective potential as a function of the condensate parameter indicated that the condensation is spontaneously generated from a zero condensate state.

The next work [14, 15], had as a main objective to justify the applicability of the Feynman expansion introduced in [13]. This search was in need because after modifying the propagator, it was unknown whether or not the initial wavefunction generating the expansion was a physical state of the free theory. In [14, 15], by making use of the operational formulation of QCD developed by Kugo and Ojima [20], it was possible to find a physical state of the non-interacting theory being able to generate through the Wick expansion, the sort of propagators considered in [13]. The discussion allowed also a more precise characterization of the class of changes admitted in the diagrammatic expansion by the physical state condition on the initial state. Specifically, it was found that the $C$ parameter, describing the gluon condensation, must be real and positive.

The present work is an expanded version of a letter sent to publication. Its main objective is to evaluate first $\left\langle G^{2}\right\rangle$ in terms of the condensate parameter $C$ up to order $g^{2}$, in order to determine the quark masses after fixing the parameter $g^{2} C$ to reproduce the accepted value for $\left\langle g^{2} G^{2}\right\rangle$. The essential result is the evaluation of the constituent masses for the light quarks. In order to perform it, we have made use of the recent values reported for the current masses [21]. The idea inspiring the work was the possibility that the gluon condensation described by modified expansion could predict the appearance of large masses for the light 
quarks $(u, d$ and $s)$ of the order of $1 / 3$ of the nucleon mass (constituent quark mass), thus furnishing an explanation for the successful constituent quark models. Surprisingly, after fixing the condensate parameter $g^{2} C$ to reproduce the most accepted value of the $\left\langle g^{2} G^{2}\right\rangle$ (through the here performed evaluation of this quantity in terms of $g^{2} C$ ), it followed that the light quarks $u, d$ and $s$ got exactly the needed constituent like values for the masses in one of the propagation modes of the modified mass shell. The free propagation modes, which are at discrepancy with the absence of free quarks, are here only arising within an approximation evaluating the main effects of the condensate. After the inclusion of all the terms completing the one loop approximation in the gluon self-energy an interesting picture arises. It follows that the previously free propagation modes of the $u$ and $d$ quarks turn to be confined. On another hand, the $s, c, b$ and $t$ quarks waves become damped. That is, the squared rest mass get complex values. It should be stressed that the peaked structure of the propagator for the $u$ and $d$ at $\sqrt{p^{2}}=m=0.30915 G e V$ signals the value of $m$ as the one expected to appear in the kinetic energy terms $\vec{p}^{2} /(2 m)$ defining the free part of the bound state equations for $u$ and $d$ constituted nucleons. Henceforth, a possible mechanism contributing to justify the drastic difference between the stability of nucleons and the higher resonances is suggested. The absence of propagating modes for the $u$ and $d$, might be assuring the absolute stability shown by the nucleons bound states. In other manner, the presence of damped modes in the rest of the quarks would contribute to the decaying channels observed for all the hadron resonances associated to these short lived states.

Next, after considering the ground state within each of the baryon resonance groups as classified in [21] and its set of three constituent quarks, and adding the calculated quark masses for each of them (which depends on the flavor through the assumed values of the current masses), a spectrum of baryon masses was estimated. The results again reproduce reasonably well the experimentally determined resonance energies [21]. This outcome suggests the less relevant role of the interaction energy among quarks in determining the masses of these hadrons.

At this point it is important to make clear that earlier works [22, 23] introduced a pure delta function at zero momentum in the gluon propagator, searching for a model of meson resonances. In these treatments the constants introduced, multiplying the delta function, were different to the one fixed in our previous work [14, 15], leading also to very different results. In particular, the singularity structure of the quark propagator have no poles on the 
real $p^{2}$ axis 23] at difference with our results. This difference is directly connected with the definite sign for the coefficient of the $\delta$-function which in our case was precisely determined in [14, 15]. Since our analysis in that paper was based in the direct construction of the state incorporating the condensate, it follows that the difference with the results of [22, 23] is rooted in a corresponding difference in the initial state determining the Wick expansion. It could be also possible that both procedures lead to the same exact physical predictions, but the first approximations in each approach could turn to be more appropriate for the discussion of specific physical issues. This question will be addressed elsewhere. It should be also further noticed that gluon condensate states reproducing the modified expansion of [22, 23] have been also discussed in [24, 25]. However, all these references are related with states showing confined quarks and massive gluons, at variance with our proposed state determining massive quarks (in the simplest approximation) and tachyonic gluons.

It is also following that another dispersion relation is obtained which predicts a vanishing value of the mass as $m_{Q} \rightarrow 0$. This mode could be connected with the family of low mass mesons (e.g. $\Pi$ and $K$ mesons). Specifically, the possibility is open that the bound states of quark excitations in these light mass states of quarks could describe such low lying mesons within the here presented framework. A circumstance pointing in this direction is that the spontaneous chirality breaking associated with this mass generation should be expected to be associated with some massless Goldstone modes. Therefore, it is possible that the bound states of such modes predicted by the Bethe-Salpeter equations turn to describe the light mesons $\Pi$ and $K$. This question however, will be deferred to a further study.

The calculations of $\left\langle G^{2}\right\rangle$ and the quark and gluon masses will be performed up order $g^{2}$. It seems worth anticipating that the approximation scheme will resemble a sort of quasiclassical limit in which the condensate seems to play a role of a macroscopic mean quantum field. Such an interpretation is in concordance with the initial motivation of our analysis as directed to construct a covariant version of the chromo-magnetic models [13.

Another conclusion which should be noticed is that the evaluated gluon mass determined from the allowed values of the condensate parameter $g^{2} C$ is tachyonic. The possibility for a tachyonic gluon mass has been recently discussed in the literature [18, 26, 27]. This result indicates that the free propagator of the expansion is not reproducing itself after the first approximation. This property, however, does not mean a limitation of the approach. This is because, at variance with [22, 23], we are not assuming that the exact propagator has the 
same delta function structure. This form only arose for the free propagator as determined by the Wick expansion around the considered initial state.

The presentation is organized as follows: Section II is devoted to make precise the elements characterizing the modified perturbation expansion. In the Section III the calculation of the $\left\langle G^{2}\right\rangle$ up to $g^{2}$ order for gluodynamics is presented and the result used to estimate the condensation parameter $g^{2} C$. Further in Section IV the effects of the condensate on the quark masses are evaluated and the obtained values used to estimate the spectrum of the ground states in each baryon group as classified in [21]. In Section V the calculation of the effect of the condensate on the one loop self-energy for the gluons is evaluated. Finally, the results are summarized and some open questions and a conjecture are underlined in the last Section VI.

\section{THE MODIFIED FEYNMAN RULES}

In this introductory section, the main elements and conventions of the modified expansion introduced in [13, 14, 15] will be reviewed. The generating functional of the Green functions in the modified theory has the form

$$
\begin{aligned}
Z[J, \bar{\eta}, \eta, \bar{\xi}, \xi] & =\frac{1}{N} \int D\left(A^{r}, \bar{c}^{r}, c^{r}, \bar{\Psi}^{r}, \Psi^{r}\right) \exp \left\{i \int d^{4} x \mathcal{L}^{\text {sources }}\right\} \\
\mathcal{L}^{\text {sources }} & =\mathcal{L}+J^{\mu, a} A_{\mu}^{r a}+\bar{c}^{r a} \eta^{a}+\bar{\eta}^{a} c^{r a}+\bar{\Psi}^{r i} \xi^{i}+\bar{\xi}^{i} \Psi^{r i} \\
N & =\int D\left(A^{r}, \bar{c}^{r}, c^{r}, \bar{\Psi}^{r}, \Psi^{r}\right) \exp \left\{i \int d^{4} x \mathcal{L}\right\}
\end{aligned}
$$

where sources for all the renormalized fields $\left(A_{\mu}^{r a}, \bar{c}^{r a}, c^{r a}, \bar{\Psi}^{r i}, \Psi^{r i}\right)$ have been introduced in the usual manner, and the effective Lagrangian for the bare fields was selected in the Kugo-Ojima quantization procedure for gauge theory as

$$
\begin{aligned}
\mathcal{L} & =\mathcal{L}_{G}+\mathcal{L}_{G h}+\mathcal{L}_{Q} \\
\mathcal{L}_{G} & =-\frac{1}{4} F_{\mu \nu}^{a}(x) F^{\mu \nu, a}(x)-\frac{1}{2 \alpha}\left(\partial^{\mu} A_{\mu}^{a}(x)\right)^{2}, \\
\mathcal{L}_{G h} & =-i \partial^{\mu} \bar{c}^{a}(x) D_{\mu}^{a b}(x) c^{b}(x), \\
\mathcal{L}_{Q} & =\bar{\Psi}^{i}(x)\left(i \gamma^{\mu} D_{\mu}^{i j}-m_{Q}^{*} \delta^{i j}\right) \Psi^{j}(x) .
\end{aligned}
$$

The sum over the six quark flavors will be omitted everywhere in order to simplify the exposition. We think that no confusion should arise for it. The gluon field intensity has the 
usual form

$$
F_{\mu \nu}^{a}(x)=\partial_{\mu} A_{\nu}^{a}(x)-\partial_{\nu} A_{\mu}^{a}(x)+g^{*} f^{a b c} A_{\mu}^{b}(x) A_{\nu}^{c}(x)
$$

where $D_{\mu}^{a b}, D_{\mu}^{i j}$ are the covariant derivatives in the adjoint and fundamental representations of the group $S U(3)$ respectively

$$
\begin{aligned}
& D_{\mu}^{a b}(x)=\partial_{\mu} \delta^{a b}-g^{*} f^{a b c} A_{\mu}^{c}(x), \\
& D_{\mu}^{i j}(x)=\partial_{\mu} \delta^{i j}-i g^{*} T^{i j, a} A_{\mu}^{a} .
\end{aligned}
$$

Here, the bare fields and bare coupling constant and masses $\left(g^{*}, m_{Q}^{*}\right)$, were expressed in terms of their renormalized counterparts following the same conventions of Ref. 28]. Below, the renormalized masses and coupling will be denoted as $m_{Q}$ and $g$ respectively.

It should be recalled that as argued in [14, 15] the physical predictions for the value $\alpha=1$ of the gauge parameter should have physical meaning whenever the adiabatic connection of the interaction does not lead the evolving state out the physical space. Therefore here we will fix this value of the parameter.

Then, after the standard procedure of extracting out of the functional integral the exponential of the terms of higher than second order in the fields (vertices) the generating functional takes the form to be employed in the evaluations below [29]:

$$
\begin{aligned}
Z[J, \bar{\eta}, \eta, \bar{\xi}, \xi]= & N^{-1} \exp \left\{i \left[\frac{S_{a b c}^{G}}{3 ! i^{3}} \frac{\delta^{3}}{\delta J_{a} \delta J_{b} \delta J_{c}}+\frac{S_{a b c d}^{G}}{4 ! i^{4}} \frac{\delta^{4}}{\delta J_{a} \delta J_{b} \delta J_{c} \delta J_{d}}\right.\right. \\
& \left.\left.+\frac{S_{r a s}^{G h}}{2 ! i^{3}} \frac{\delta^{3}}{\delta \bar{\eta}_{r} \delta J_{a} \delta\left(-\eta_{s}\right)}+\frac{S_{i a j}^{Q}}{i^{3}} \frac{\delta}{\delta \bar{\xi}_{i} \delta J_{a} \delta\left(-\xi_{j}\right)}\right]\right\} Z_{0}[J, \bar{\eta}, \eta, \bar{\xi}, \xi],
\end{aligned}
$$

with the free functional given by

$$
Z_{0}[J, \bar{\eta}, \eta, \bar{\xi}, \xi]=\exp \left\{i \frac{J_{a} G_{G}^{a b} J_{b}}{2}+i \bar{\eta}_{r} G_{G h}^{r s} \eta_{s}+i \bar{\xi}_{i} G_{Q}^{i j} \xi_{j}\right\}
$$

and $N$ normalizes $Z$ to be one at vanishing sources. Use will be made of the DeWitt compact notation [30, in which a Latin letter let say $a, b, \ldots$, for a field symbolizes the space-time coordinates of it as well as all its internal quantum numbers. The same index in a source or a tensor symbolizes the same set of variables of the kind of fields associated with this specific index. For example $A_{a}=A_{\mu_{a}}^{a}\left(x_{a}\right), c_{a}=c^{a}\left(x_{a}\right)$ and $\Psi^{i}=\Psi^{i}\left(x_{i}\right)$. Note that a particular convention has been also employed in which the same letter is employed for the global index (for all the coordinated and internal indices) and the one indicating the internal coordinates 
in the explicit form of the quantities. Such a procedure was useful for the calculations and we also think that will not create confusion. As usual, repeated indices represent the corresponding space time integrals and contracted, Lorentz, spinor or color components.

The following definitions also have been used in (8):

$$
\begin{array}{rlrl}
S_{i j k \ldots}^{\alpha} & \equiv\left(\frac{\delta}{\delta \Phi_{i}} \frac{\delta}{\delta \Phi_{j}} \frac{\delta}{\delta \Phi_{k}} \ldots S^{\alpha}(\Phi)\right)_{\Phi=0} \quad \text { for } \alpha=G, G h, Q \text { and } \Phi=A, \bar{c}, c, \bar{\Psi}, \Psi \\
G_{\alpha}^{i j} & \equiv-S_{\alpha, i j}^{-1} & \text { for } \alpha=G, G h, Q
\end{array}
$$

where $G, G h$ and $Q$ mean the gluon, ghost and quark parts of the action respectively.

There is only one main element determining the difference of the usual Perturbative QCD expansion and the modified one considered in [13, 14, 15]. It is related with the form of the gluon free propagator. As proposed in [13] there is an additional term to the gluon free propagator which is absent in the standard expansion. In [14, 15], such a term was shown to be a consequence of a Wick expansion based in a state constructed by acting on the usual vacuum with an exponential of pairs of zero momentum gluon and ghost creation operators. The ghost propagator [14, 15] could remain unmodified if the parameter left after fixing the form of the wave-function is taken as a real and positive one [14, 15]. Such a selection will be employed here. The quark propagator is not affected in any way by the gluon condensation as introduced in [13, 14, 15]. However, as it will be commented in the summary, the results of the present work led to the idea about a possible physical relevance of introducing quark condensates along the similar lines as it was done for the gluons. We consider the exploration of this possibility to be exposed as one of the most interesting extensions of the work.

A technical point can be noticed for precision. The operator quantization employed in [14, [15] takes the ghost fields as satisfying the conjugation properties proper of the approach of Kugo and Ojima [20]. However, at the level of the Feynman diagram expansion the difference with the standard procedure is only a change of variables.

In accordance with above remarks and the results of [13, 14, 15] the diagram technique defining the modified expansion has the following basic propagators for the renormalized 
fields

$$
\begin{aligned}
G_{G}^{a b} & =\delta^{a b} g^{\mu_{a} \mu_{b}}\left[\frac{1}{k^{2}+i \varepsilon}-i C \delta(k)\right], \\
G_{G h}^{r s} & =\delta^{r s} \frac{(-i)}{k^{2}+i \varepsilon} \\
G_{Q}^{i j} & =\delta^{i j} \frac{m+p^{\mu} \gamma_{\mu}}{\left(m^{2}-p^{2}-i \varepsilon\right)} .
\end{aligned}
$$

and the same normal and counterterm vertices of the usual theory 28]. The dimensional regularization and minimal substraction (MS) is the renormalization procedure employed here.

Being defined the Feynman rules and the conventions to be used, below they will be applied to the calculation of various quantities of interest.

\section{THE MEAN VALUE OF $G^{2}$}

The calculation of the mean value of the gluon field intensity squared is considered in this section. It extends the former evaluation done in [13] within a simpler approximation. The result will be employed afterwards for fixing the value of the condensation parameter $g^{2} C$ to be the necessary one for furnishing the currently estimated value for $\left\langle g^{2} G^{2}\right\rangle$. The calculation will be performed up to order $g^{2}$ including all the dependence on the condensate.

It is a helpful circumstance here, that the one loop renormalization procedure of the modified expansion can be performed identically as in the standard PQCD. This is a direct consequence of the fact that the $\delta$-functions kill the only existing integrals at the one loop level and therefore all the corresponding counterterms in the dimensional regularization are identical. This fact will be helpful in regularizing and renormalizing the composite operator $\left\langle G^{2}\right\rangle$.

For the evaluation of $\left\langle G^{2}\right\rangle$ the following definition of this quantity will be employed

$$
\left\langle G^{2}\right\rangle=\frac{\left\langle 0\left|S_{g}\right| 0\right\rangle}{\int d x^{D}}=\frac{\int D\left(A^{r}, \bar{c}^{r}, c^{r}, \bar{\Psi}^{r}, \Psi^{r}\right) S_{g}\left[A^{r}\right] \exp \left\{i \int d^{4} x \mathcal{L}^{\text {sources }}\right\}}{\int d x^{D} \int D\left(A^{r}, \bar{c}^{r}, c^{r}, \bar{\Psi}^{r}, \Psi^{r}\right) \exp \left\{i \int d^{4} x \mathcal{L}\right\}},
$$

where $S_{g}\left[A^{r}\right]$ represents the gluon part of the action in the absence of the gauge breaking term depending on the gauge parameter $\alpha$, evaluated at the renormalized gluon fields $A^{r}$, that is

$$
S_{g}\left[A^{r}\right]=-\frac{1}{4} \int d^{4} x F_{\mu \nu}^{r a}(x) F^{r \mu \nu, a}(x) .
$$


Therefore, $\left\langle G^{2}\right\rangle$ will be considered as defined by the mean value of the gluon action divided by the space-time volume in dimensional regularization.

After substituting in (9) the perturbative expression for the mean value in terms of the Generating Functional (8) it follows

$$
\left\langle 0\left|S_{g}\right| 0\right\rangle=\left\{\left[\frac{S_{a b}^{g}}{2 i^{2}} \frac{\delta^{2}}{\delta J_{a} \delta J_{b}}+\frac{S_{a b c}^{g}}{3 ! i^{3}} \frac{\delta^{3}}{\delta J_{a} \delta J_{b} \delta J_{c}}+\frac{S_{a b c d}^{g}}{4 ! i^{4}} \frac{\delta^{4}}{\delta J_{a} \delta J_{b} \delta J_{c} \delta J_{d}}\right] Z[J, \bar{\eta}, \eta, \bar{\xi}, \xi]\right\}_{J, \bar{\eta}, \eta, \bar{\xi}, \xi=0} .
$$

In the momentum representation the kernel $S_{a b}^{g}$ has the form

$$
S_{a b}^{g}=(2 \pi)^{4} \delta\left(k_{a}+k_{b}\right) \delta^{a b}\left[-\left(g_{\mu_{a} \mu_{b}} k_{a}^{2}-k_{a, \mu_{a}} k_{a, \mu_{a}}\right)\right]
$$

and the $S_{a b c}^{g}$ and $S_{a b c d}^{g}$ ones are the usual three and four legs gluon vertices [28].

Let us consider below the evaluation of $\left\langle G^{2}\right\rangle$ up to order $g^{2}$. As any Feynman diagram can be expressed as a polynomial in the constant $C$, after associating a new diagrammatic line for this part of the free propagator, all the Feynman diagrams can be divided in two groups. The first, let say $G_{1}$ including the diagrams not showing the new propagator line and another group, $G_{2}$ having one or more, let us call them, "condensate" lines. Such lines have associated to them a multiplicative Dirac delta function at zero momentum.

After writing out all the expressions of type $G_{2}$ (up to second order in the renormalized coupling $g^{2}$ ) contributing to (10), it follows that the only non-vanishing diagram is the one appearing in the Figure 1 in which both gluon lines are substituted by "condensate" lines. The remaining terms vanish thanks to the zero momentum evaluation produced by the Dirac Delta functions introduced by the "condensate" lines and also due to the vanishing of tadpoles in dimensional regularization. Also, it should be underlined that the retention of the $i \epsilon$ factors defining the Feynman propagator, up to the evaluation of the integrals was also important in obtaining the zero result for some of the diagrams in $G_{2}$.

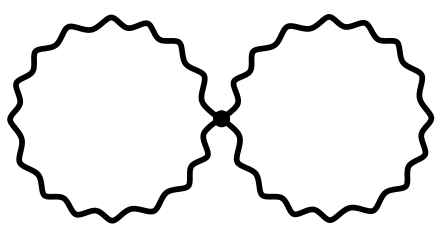

FIG. 1: Diagram contributing to $G_{2}$ terms in the gluon condensate parameter. 
The contribution related with Figure 1 had been calculated before and the result is [13, 14]

$$
T_{G_{2}}=-\frac{1}{4} \frac{288 g^{2} C^{2}}{(2 \pi)^{8}} .
$$

Next, the diagrams in $G_{1}$ are the ones contributing to $G^{2}$ up to order $g^{2}$ in standard perturbative QCD. They are illustrated in Figure 2. The terms $T_{6}$ and $T_{7}$ represent the only non-vanishing and non-tadpole like second order contributions. It can be noticed that the appearance of two tadpoles in the term $T_{7}$ leads to the exact vanishing of this contribution in dimensional regularization.

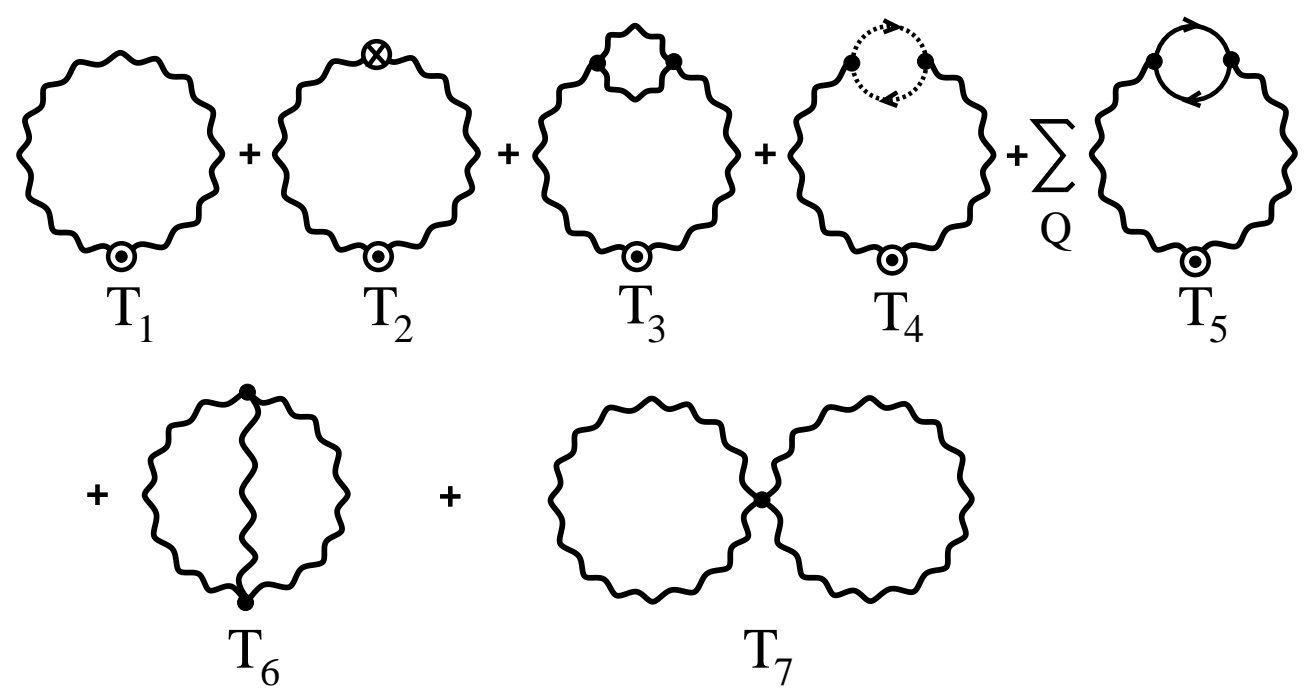

FIG. 2: Diagram contributing to $G_{1}$ terms in the gluon condensate parameter.

For the diagram $T_{6}$ corresponds the expression

$$
E_{T_{6}}=\frac{1}{3 ! i^{3}} \int \frac{d p^{D}}{(2 \pi)^{D} i} \frac{g^{\mu \nu} \delta^{a b}}{p^{2}+i \varepsilon} \Pi_{\mu \nu}^{(g)}{ }^{a b}(p),
$$

where $\Pi^{(g)}$ is the gluonic contribution to the polarization tensor in second order, which has been explicitly calculated. After substituting the known result for $\Pi$ and performing the Wick rotation, the previous expression turns out to be of the form

$$
E_{T_{6}} \backsim \int \frac{d q^{D}}{(2 \pi)^{D}}\left(q^{2}\right)^{\frac{D}{2}-2}
$$

Then considering the formula 31]

$$
\begin{aligned}
\int \frac{d q^{D}}{(2 \pi)^{D}} \frac{1}{\left(q^{2}\right)^{\alpha}} \frac{1}{(q-k)^{2 \beta}} & =\frac{i(-1)^{-\alpha-\beta}\left(-k^{2}\right)^{-\alpha-\beta+n / 2}}{\left(16 \pi^{2}\right)^{n / 4}} \frac{\Gamma(\alpha+\beta-n / 2)}{\Gamma(\alpha) \Gamma(\beta)} B\left(\frac{n}{2}-\beta, \frac{n}{2}-\alpha\right), \\
B(n, m) & =\frac{\Gamma(m) \Gamma(n)}{\Gamma(m+n)},
\end{aligned}
$$


for the case $\alpha+\beta=2-\frac{D}{2}$ and fixing $k_{\mu}=0$, it follows the vanishing of diagram $T_{6}$ within the dimensional regularization for $D>2$.

It rest now to discuss the contributions of the tadpole like diagrams. It is worth noticing at this point that they are quite similar in their form to the one corresponding to the square of the electromagnetic field strength in QED. In fact they differ from it only in some numerical color factors. An important quantitative difference between the expressions for both theories is related with magnitude of the coupling constant which is very much higher in QCD. Let us consider the $T_{1}-T_{5}$ terms in Figure 2 embodying the gluon, ghost and fermion contributions with the inclusion of the one loop counterterms rendering finite the polarization operator at one loop [28]. This expression turns to be of the form

$$
E_{T_{1}-T_{5}} \backsim \int \frac{d p^{D}}{(2 \pi)^{D}} \frac{\left(p_{\mu} p_{\nu}-p^{2} g_{\mu \nu}\right)\left(g^{\mu \nu}-\frac{p^{\mu} p^{\nu}}{p^{2}+i \varepsilon}\right)}{p^{2}+i \varepsilon} \delta^{a a}\left(1-\Pi\left(p^{2}\right)\right),
$$

where $\Pi$ as usual is defined through the polarization tensor

$$
\Pi_{\mu \nu}^{a b}(p)=\delta^{a b}\left(p_{\mu} p_{\nu}-p^{2} g_{\mu \nu}\right) \Pi\left(p^{2}\right)
$$

and it can be expressed as the superposition of the pure gluodynamical contribution and the quark one as 28]

$$
\begin{aligned}
\Pi\left(p^{2}\right)= & \Pi_{G}\left(p^{2}\right)+\Pi_{Q}\left(p^{2}\right) \\
= & \frac{g^{2} \mu^{4-D}}{4 \pi^{D / 2}}\left(-p^{2}\right)^{2-D / 2} \Gamma\left(2-\frac{D}{2}\right) B\left(\frac{D}{2}, \frac{D}{2}\right) \times \\
& \times\left(-\frac{C_{G}(3 D-2)}{\frac{D}{2}-1}+\frac{8 T_{R}}{B\left(\frac{D}{2}, \frac{D}{2}\right)} \sum_{Q} \int_{0}^{1} d x x(1-x)\left(x(1-x)-\frac{m_{Q}^{2}-i \varepsilon}{p^{2}}\right)^{-(2-D / 2)}\right) \\
& +\left(Z_{3}-1\right),
\end{aligned}
$$

where $C_{G}=3$ and $T_{R}=1 / 2$ for $S U(3)$ and $\mu$ is the scale mass in the dimensional regularization.

Considering first the pure gluonic contributions associated $\left(1-\Pi_{G}\left(p^{2}\right)\right)$ in (12), it follows that they reduce to a sum of dimensional regularized integrals of the same form as (11). Thus, the second order gluodynamical contribution of the tadpole like contributions vanish. Let us analyze now the quark contributions associated to $\Pi_{Q}$. It can be noticed that for massless quarks, that is $m_{Q}=0$, the $\Pi_{Q}$ has the same analytical dependence of $p^{2}$ as the gluonic part $\Pi_{G}$ and all the quark contributions vanish after the regularization is employed. However, for 
massive quarks the result for the second order dimensionally regularized contribution along the same lines employed for the previous terms gives rise to a non-vanishing contribution of the following form after the substraction of the divergent pole terms at $D=4$. The expression for the regularized term before substraction is

$$
g^{2} \mu^{4-D} E_{T_{6}}=\left(\sum_{Q}\left(m_{Q}^{2}\right)^{D-2}\right) \frac{64 \sqrt{\pi} g^{4} \mu^{8-2 D} T_{R}(D-1) \Gamma(2-D) \Gamma(4-D)}{2^{7-2 D}(4 \pi)^{D} \Gamma\left(\frac{9}{2}-D\right)} .
$$

This expression has simple as well as double poles which after substracted (in the $D=$ 4 limit following the rules of the MS scheme for composite operators [32]) give a finite correction to $\left\langle g^{2} G^{2}\right\rangle$ for the gluon condensate parameter. It can be evaluated and the numerical expression becomes

$$
\left(g^{2} \mu^{4-D} E_{T_{6}}\right)^{f i n}=0.00064 T_{R} g^{4}\left(\sum_{Q} m_{Q}^{4}\left[55.32-37.4 \ln \left(\frac{\mu^{2}}{m_{Q}^{2}}\right)+6.0 \ln ^{2}\left(\frac{\mu^{2}}{m_{Q}^{2}}\right)\right]\right) .
$$

However, it should be noticed that this finite expression has been obtained for the second order correction of the polarization operator. The summation of the infinite ladder of self-energy insertions leading to the one loop propagator can be suspected to produce a noticeable modification of this result. After also considering the high indefiniteness in the quark current masses, and the technical difficulty we had in evaluating the dimensionally regularized expression for the above mentioned self-energy insertions, we decided to employ for the further consideration here the purely gluodynamical contribution. In support of this way of proceeding there is also another circumstance. It is needed to notice that a refined version of the definition of the composite operator $G^{2}(x)$ could be suspected to exist in such a way that the terms associated to a tadpole diagram of the exact propagator vanish. This property would assure the exact vanishing of the $\left\langle F^{2}\right\rangle$ in $\mathrm{QED}$ a property that to our knowledge, is expected. Because, at one loop, both QED and QCD tadpole expressions are basically the same, the above written quark contribution would not really appear. The existence of the mentioned definition, opens the possibility for the second order gluodynamical contribution to be exact one for QCD in this approximation. However, we shift the discussion of this question to further analysis to be considered elsewhere and simply adopt the gluodynamical result.

Therefore, the total second contribution to the mean value of $G^{2}$ in gluodynamics is 
defined by the only non-vanishing term

$$
\left\langle G^{2}\right\rangle=\frac{288 g^{2} C^{2}}{(2 \pi)^{8}}
$$

coming from the condensate contributions arising from the diagram $T_{6}$ which is defined by the four gluon interactions. Finally, let us determine in a phenomenological way a value for the constant $g^{2} C$ by making use of a currently estimated value for $\left\langle g^{2} G^{2}\right\rangle$ :

$$
\left\langle g^{2} G^{2}\right\rangle \cong 0.5\left(G e V / c^{2}\right)^{4}
$$

Therefore, the parameter $g^{2} C$ takes the value:

$$
g^{2} C=64.9394\left(G e V / c^{2}\right)^{2}
$$

This fixing of the condensation parameter allows to investigate the physical predictions of the modified expansion in the next section.

\section{CONSTITUENT QUARKS MASSES}

Let us consider now the effect on the current quark masses produced by the condensate. The full inverse propagator for the quarks in terms of the corresponding mass operator has the general expression

$$
G_{Q}^{-1}(p)=\left(m_{Q}-p^{\mu} \gamma_{\mu}-\Sigma(p)\right)
$$

where the color index identity matrix has been omitted and $\Sigma(p)$ is the mass operator. Its expression is related with the diagram in Figure 3.

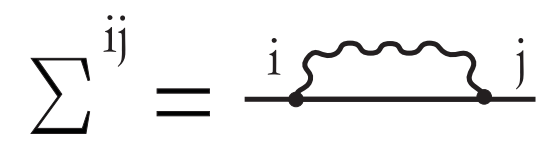

FIG. 3: Diagram for $\Sigma(p)$ in one loop.

The form for $\Sigma(p)$ up to order $g^{2}$, within the modified Feynman rules can be written as

$$
\Sigma(p)=g^{2} C_{F} \int \frac{d^{4} k}{(2 \pi)^{4} i} \frac{\gamma_{\mu}\left(m_{Q}+(p-k)^{\alpha} \gamma_{\alpha}\right) \gamma_{\nu} G_{G}^{\mu \nu}(k)}{\left(m_{Q}^{2}-(p-k)^{2}\right)}
$$

in which the color factor $C_{F}$ for the $S U(N)$ group is $C_{F}=\frac{N^{2}-1}{2 N}$ which for the case of interest here $S U(3)$, reduces to $C_{F}=\frac{4}{3}$ [28]. 
Taking into account the modified gluon propagator and the standard relations for the $\gamma$ matrices (which here are adopted in the same way as in Ref. [28]) the one loop mass operator expression is simplified to be

$$
\Sigma(p)=g^{2} C_{F} \int \frac{d^{4} k}{(2 \pi)^{4} i} \frac{2\left(2 m_{Q}-(p-k)^{\alpha} \gamma_{\alpha}\right)}{\left(m_{Q}^{2}-(p-k)^{2}\right)}\left(\frac{1}{k^{2}}-i C \delta(k)\right) .
$$

Let us disregard for the moment the term not involving the condensate parameter $C$ in order to first study the sole influence on the quark masses of the condensation effect. Then, the expression for $\Sigma$ reduces to

$$
\Sigma(p)=-\frac{g^{2} C_{F} C}{(2 \pi)^{4}} \frac{2\left(2 m_{Q}-p^{\alpha} \gamma_{\alpha}\right)}{\left(m_{Q}^{2}-p^{2}\right)} .
$$

Therefore, the inverse propagator for the quark takes the simple form

$$
G_{Q}^{-1}(p)=m_{Q}\left(1+2 \frac{M^{2}}{\left(m_{Q}^{2}-p^{2}\right)}\right)-p^{\mu} \gamma_{\mu}\left(1+\frac{M^{2}}{\left(m_{Q}^{2}-p^{2}\right)}\right),
$$

where the constant $M$ is defined by

$$
M^{2}=\frac{2 g^{2} C_{F} C}{(2 \pi)^{4}}=0.1111\left(G e V / c^{2}\right)^{2},
$$

in which the numerical value has been obtained by using (14).

The zeros of the determinant associated to the inverse propagator (17) then allows to determine the effects of the condensate (reflected by the parameter $M$ ), on the effective mass of the quarks. Let below $s, s=1 \ldots 6$ an index indicating each kind of quark characterized by its particular current mass $m_{Q_{s}}$.

In order to find the dependence of the effective quark mass $m_{q_{s, i}}^{2}$ as a function of the current mass parameters $m_{Q_{s}}$ an analysis was done for the zeros of the determinant of the inverse green function (17). It follows that there is only one solution having a squared mass being positive for arbitrary values of the current quark masses. The existence of this real and positive solution for the squared mass becomes possible thanks to the real and positive character of $C$, as was shown previously [14, 15]. There is another interesting branch of the zeros of the determinant (dispersion relations) showing vanishing values for the effective mass in the light current mass limit. For the purely real solution the value of the effective quark mass $m_{q_{s}}=f\left(m_{Q_{s}}\right)$ as a function of $m_{Q_{s}}$ is shown in the Figure . The graph is plotted for the region $m_{Q_{s}}<2 \mathrm{GeV} / \mathrm{c}^{2}$ which contains the current mass values of the $u, d, s$ and $c$ quarks. 


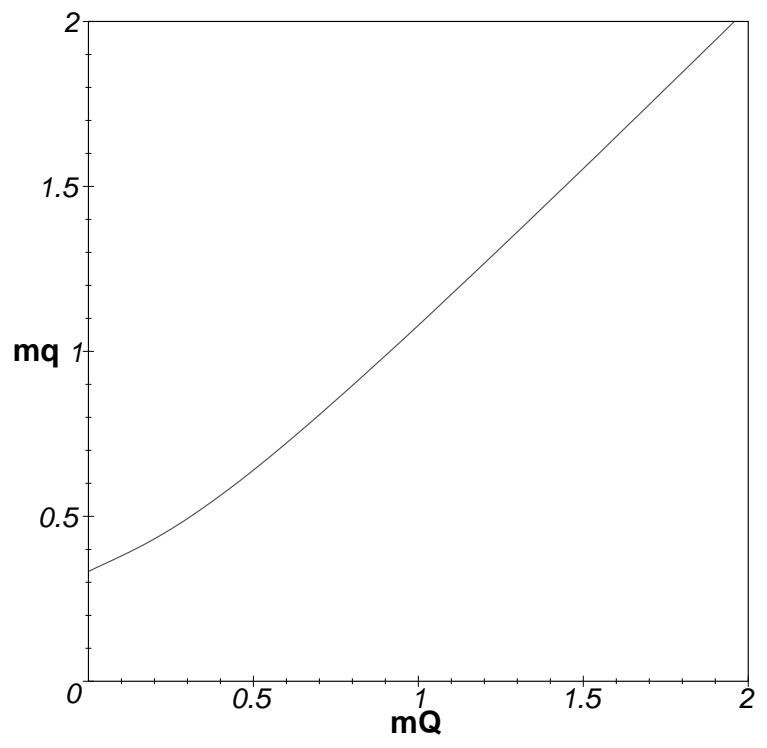

FIG. 4: Real solution for the quark mass as a function of the Lagrangian mass (masses in $G e V / c^{2}$ ).

As it can be appreciated in the picture, the calculated effective quark masses for light flavors $(u, d$ and $s)$ are clearly predicting the values of the quark masses being in use in the constituent quark models of the baryons. That is, the light quarks get a weight of near one third of the nucleon mass. In the table [ the mass values obtained for each of the quark flavors are shown [21]. From the global properties of table —it can be observed that the main effect of the gluon condensate seems to be the dressing the light quarks with a cloud gluons having a total mass of one third of the nucleon mass. Then, the results point in the direction of the mainly glue nature of the constituent quark masses of the $u, d$ and $s$ quarks within many baryon resonance. These results then support the idea that a modified perturbative expansion like the one being considered, in which the effect of the gluon condensate have been incorporated, would be able to predict with reasonably good approximation properties of the low energy strong interactions. Similar values for the constituent quarks masses have been obtained by different methods in [33, 34].

We will now investigate the influence on the mass spectrum produced by including the standard one loop contribution in the dispersion relations. After substituting the known 31 quark one-loop self-energy expression, the one loop inverse quark propagator can be written 
TABLE I: Quark mass values in presence of the condensate in units of $M e V / c^{2}$.

\begin{tabular}{||c||c||c||c||}
\hline \hline Quarks & $m_{\text {Low }}^{\text {Exp }}$ & $m_{\text {Up b }}^{\text {Exp }}$ & $m_{\text {Med }}^{\text {Theo }}$ \\
\hline \hline$(\mathrm{u})$ & 1.5 & 5 & 334.944 \\
\hline \hline$(\mathrm{d})$ & 3 & 9 & 336.287 \\
\hline \hline$(\mathrm{s})$ & 60 & 170 & 388.191 \\
\hline \hline$(\mathrm{c})$ & 1100 & 1400 & 1315.241 \\
\hline \hline$(\mathrm{b})$ & 4100 & 4400 & 4269.572 \\
\hline \hline$(\mathrm{t})$ & 168600 & 179000 & 173800.48 \\
\hline \hline
\end{tabular}

${ }^{a}$ Reported lower bound value for the Lagrangian mass

${ }^{b}$ Reported upper bound value for the Lagrangian mass

${ }^{c}$ Calculated mean value of the constituent mass, for the lower and upper bound of the Lagrangian masses as determined only by the condensate contribution to the self-energy in the one loop approximation

as

$$
\begin{aligned}
G_{Q}^{-1}(p)= & \left(m_{Q}-p^{\mu} \gamma_{\mu}-\Sigma(p)\right)=A\left(p^{2}\right) m_{Q}-B\left(p^{2}\right) p^{\mu} \gamma_{\mu} \\
= & m_{Q}\left[1+\frac{4 C_{F} g^{2} C}{(2 \pi)^{4}} \frac{1}{m_{Q}^{2}-p^{2}}-\frac{C_{F} g^{2}(\mu)}{(4 \pi)^{2}} \times\right. \\
& \left.\left(-6+4 \gamma-\ln (4 \pi)+4 \ln \left(\frac{m_{Q}^{2}-p^{2}}{\mu^{2}}\right)-4 \frac{m_{Q}^{2}}{p^{2}} \ln \left(\frac{m_{Q}^{2}-p^{2}}{m_{Q}^{2}}\right)\right)\right] \\
& -p^{\mu} \gamma_{\mu}\left[1+\frac{2 C_{F} g^{2} C}{(2 \pi)^{4}} \frac{1}{m_{Q}^{2}-p^{2}}+\frac{C_{F} g^{2}(\mu)}{(4 \pi)^{2}} \times\right. \\
& \left.\left(1-\gamma+\ln (4 \pi)+\frac{m_{Q}^{2}}{p^{2}}-\ln \left(\frac{m_{Q}^{2}-p^{2}}{\mu^{2}}\right)+\left(\frac{m_{Q}^{2}}{p^{2}}\right)^{2} \ln \left(\frac{m_{Q}^{2}-p^{2}}{m_{Q}^{2}}\right)\right)\right],
\end{aligned}
$$

where the dimensionless coupling constant $g^{2}$ has been substituted by its running expression as a function of the scale parameter $\mu$ as

$$
g(\mu)^{2}=\frac{4 \pi 0.12}{1+\frac{70.24}{4 \pi} \ln \left(\frac{\mu}{91.18}\right)} .
$$

That is, the value of $\alpha^{2}(\mu)=g^{2}(\mu) /(4 \pi)$ is fixed to be 0.12 at the scale of the $Z$ mass $\mu=m_{Z}=91.18 \mathrm{GeV}$. In order to automatically fix the scale $\mu$ to the one associated to the magnitude of the obtained solution for the mass, the value of $\mu$ was taken to satisfy $\mu^{2}=p^{2}$. That is, to reproduce the same value of the solution for the squared mass. 
Before proceeding, it should be noticed that the inverse propagator, after the introduction of the one loop terms, gets a branch cut at the real axes for the considered complex variable $p^{2}$ for $\operatorname{Re}\left(p^{2}\right)>m_{Q}^{2}$. However, the $i \epsilon$ prescription selects one of the sides of the cut as giving a well defined value of the inverse propagator at real $p^{2}$ values.

After multiplying (18) by $A\left(p^{2}\right) m_{Q}-B\left(p^{2}\right) p^{\mu} \gamma_{\mu}$ the modified mass shells can be most easily found by solving

$$
D\left(q^{2}\right)=A^{2}\left(p^{2}\right) m_{Q}^{2}-B^{2}\left(p^{2}\right) p^{2}=0 .
$$

Concretely, we estimated the corrected values of the masses in Table $[$ by searching for the peaks of the inverse for the absolute value of $D\left(q^{2}\right)$ as a 2D-function of the real and imaginary parts of $p^{2}$. The new values are reported in Table [I]. As it can be observed the masses were not drastically changed. They all show a relatively small reduction in their magnitude, then indicating the consistency of the predicted constituent masses for the light quarks at the one loop level.

TABLE II: Quark mass values in presence of the condensate in units of $M e V / c^{2}$.

\begin{tabular}{||c||c||c||c||}
\hline \hline Quarks & $m_{\text {Low }}^{\text {Exp }}$ & $m_{U p}^{E x p} b$ & $m_{M e d}^{\text {Theo }}$ \\
\hline \hline$(\mathrm{u})$ & 1.5 & 5 & 309.15 \\
\hline \hline$(\mathrm{d})$ & 3 & 9 & 309.15 \\
\hline \hline$(\mathrm{s})$ & 60 & 170 & 354.00 \\
\hline \hline$(\mathrm{c})$ & 1100 & 1400 & $1343-200 i$ \\
\hline \hline$(\mathrm{b})$ & 4100 & 4400 & $4515-65 i$ \\
\hline \hline$(\mathrm{t})$ & 168600 & 179000 & $190000-1450 i$ \\
\hline \hline
\end{tabular}

${ }^{a}$ Reported lower bound value for the Lagrangian mass

${ }^{b}$ Reported upper bound value for the Lagrangian mass

${ }^{c}$ Calculated mean value of the constituent mass, for the lower and upper bound of the Lagrangian masses after the inclusion of the standard one loop correction for the self-energy.

An interesting outcome following from the inclusion of the standard one loop terms, should be stressed. It consists, in that for the smallest $m_{Q}$ masses associated to the lower and higher bounds for the $u$ and $d$ quarks, $\frac{1}{\left|D\left(q^{2}\right)\right|}$ although showing a definite peak at $0.30915 \mathrm{GeV}$ does not really diverge near this value. Therefore, the propagator is not 
showing a definite pole associated to a propagating quark mode at this $p^{2}$ value. However, at the level of the Bethe-Salpeter equation for bound states of quarks, it should be expected that $p^{2}$ at this peak should behave as the non-relativistic value for the square of the mass entering the kinetic energy expression $\frac{p^{2}}{2 m_{Q}}$. Henceforth, in spite of the presence of free propagating modes in the simplest approximation, the inclusion of the quantum one loop correction makes the $u$ and $d$ modes non-propagating (confined) in the same way as it occurs in the approach of Munczek and Nemirovsky [22, 23].

For greater values of $m_{Q}$ in the range presently estimated for the $s$ quark current mass $(0.060-0.170 \mathrm{GeV})$ in the neighborhood of the non-diverging peak of $\frac{1}{\left|D\left(q^{2}\right)\right|}$, this quantity starts showing divergency points at complex values of $p^{2}$ which represent damped but propagating quark modes. The just described structure of the spectrum suggests a possible explanation for the drastic difference of stability between the $u$ and $d$ composed hadrons and the resonances including $s, c, b$ and $t$ quarks. The point is that the presence of poles at complex $p^{2}$ for the heavier quarks could lead to the existence of disintegration channels for the higher mass resonances in which the $s, c, b$ and $t$ quarks can escape in a damped propagating mode to be after hadronized by some separate mechanism. On another hand, as the $u$ and $d$ quarks do not show propagating states, that could remain in a perennial confinement within an undisturbed nucleon. A further study of the consistency of this explanation is expected to be done within the planned extension of this work to investigate bound state equations for hadrons.

It can be concluded that the constituent quark masses evaluated from the simplest approximation for the dispersion relation considered above are stable under the inclusion of the standard one loop corrections to the self-energy.

Next, in the Table [II, the masses of the ground state resonance selected each of them from one of the groups of baryons as classified in [21], are shown in comparison with the values obtained for them by a theoretical estimate consisting in adding the here calculated masses for each of their known quark constituents. The quark masses of the Table [ were employed for this evaluation. Also, in the Table IV, the masses for some vector mesons reported also in [21] are shown in conjunction with the result of the sum of the calculated masses for their constituent quarks (Table [).

As it can be appreciated the results obtained for the vector mesons and baryon resonance are only estimates. However, the obtained values are in a reasonable well correspondence 
TABLE III: Experimental and Theoretical Baryonic Resonance Masses in units of $\mathrm{MeV} / \mathrm{c}^{2}$.

\begin{tabular}{||c||r||r||r||}
\hline \hline Baryon & Exp.Val. & Th.Mean.Val. & Rel.Err. \\
\hline \hline $\mathrm{p}(\mathrm{uud})$ & 938.27231 & 1006.175 & 7.24 \\
\hline \hline $\mathrm{n}(\mathrm{udd})$ & 939.56563 & 1007.519 & 7.23 \\
\hline \hline$\Lambda^{(\mathrm{uds})}$ & 1115.683 & 1059.422 & 5.04 \\
\hline \hline$\Sigma^{+}(\mathrm{uus})$ & 1189.37 & 1058.078 & 11.04 \\
\hline \hline$\Sigma^{0}(\mathrm{uds})$ & 1192.642 & 1059.422 & 11.17 \\
\hline \hline$\Sigma^{-}(\mathrm{dds})$ & 1197.449 & 1060.766 & 11.41 \\
\hline \hline$\Xi^{0}(\mathrm{uss})$ & 1314.9 & 1111.325 & 15.48 \\
\hline \hline$\Xi^{-}(\mathrm{dss})$ & 1321.32 & 1112.669 & 15.79 \\
\hline \hline$\Omega^{-}(\mathrm{sss})$ & 1642.45 & 1164.572 & 29.10 \\
\hline \hline$\Lambda_{c}^{+}(\mathrm{udc})$ & 2284.9 & 1986.472 & 13.07 \\
\hline \hline$\Xi_{c}^{+}(\mathrm{usc})$ & 2465.6 & 2038.375 & 17.33 \\
\hline \hline$\Xi_{c}^{0}(\mathrm{dsc})$ & 2470.3 & 2039.719 & 17.43 \\
\hline \hline$\Omega_{c}^{0}(\mathrm{ssc})$ & 2704 & 2091.622 & 22.65 \\
\hline \hline$\Lambda_{b}^{0}(\mathrm{udb})$ & 5624 & 4940.803 & 12.15 \\
\hline \hline
\end{tabular}

TABLE IV: Experimental and Theoretical Masses for a group of Vector Mesons in units of $M e V / c^{2}$.

\begin{tabular}{||r||r||r||r||}
\hline \hline Meson & Exp.Val. & Th.Mean.Val. & Rel.Err. \\
\hline \hline$\rho\left(\frac{u \bar{u}-d \bar{d}}{\sqrt{2}}\right)$ & 770.0 & 671.231 & 12.83 \\
\hline \hline$\varpi\left(\frac{u \bar{u}+d \bar{d}}{\sqrt{2}}\right)$ & 781.94 & 671.231 & 14.16 \\
\hline \hline$\phi(s \bar{s})$ & 1019.413 & 776.381 & 23.84 \\
\hline \hline$J / \psi(1 S)(c \bar{c})$ & 3096.88 & 2630.482 & 15.06 \\
\hline \hline$Y(1 S)(b \bar{b})$ & 9460.37 & 8539.144 & 9.74 \\
\hline \hline
\end{tabular}

with the reported experimental values. It can be also noticed that the validity of the present calculations implies that the binding energy contribution to the baryon rest masses should be small. This property then could justify a non relativistic description of such resonances. 
As it was remarked before, another form of the dependence of the effective quark mass with $m_{Q_{s}}$ is also following from the dispersion relations. It, by contrast shows a vanishing effective mass when $m_{Q_{s}} \rightarrow 0$. The existence of this branch opens the possibility for the existence an appropriate description of the light mesons ( $\Pi$ and $K$ ) within the proposed scheme. They could be associated to bound states of light quarks propagating in such modes. The analysis of this question deserves a separate treatment to be examined elsewhere. The graphic for the mentioned branch is represented in Figure 5, for the region of $m_{Q_{s}}<$ $0.1 \mathrm{GeV} / \mathrm{c}^{2}$, a point at which the change in the analytic dependence signals where the solution gets an imaginary part when the current mass value $m_{Q}$ grows.

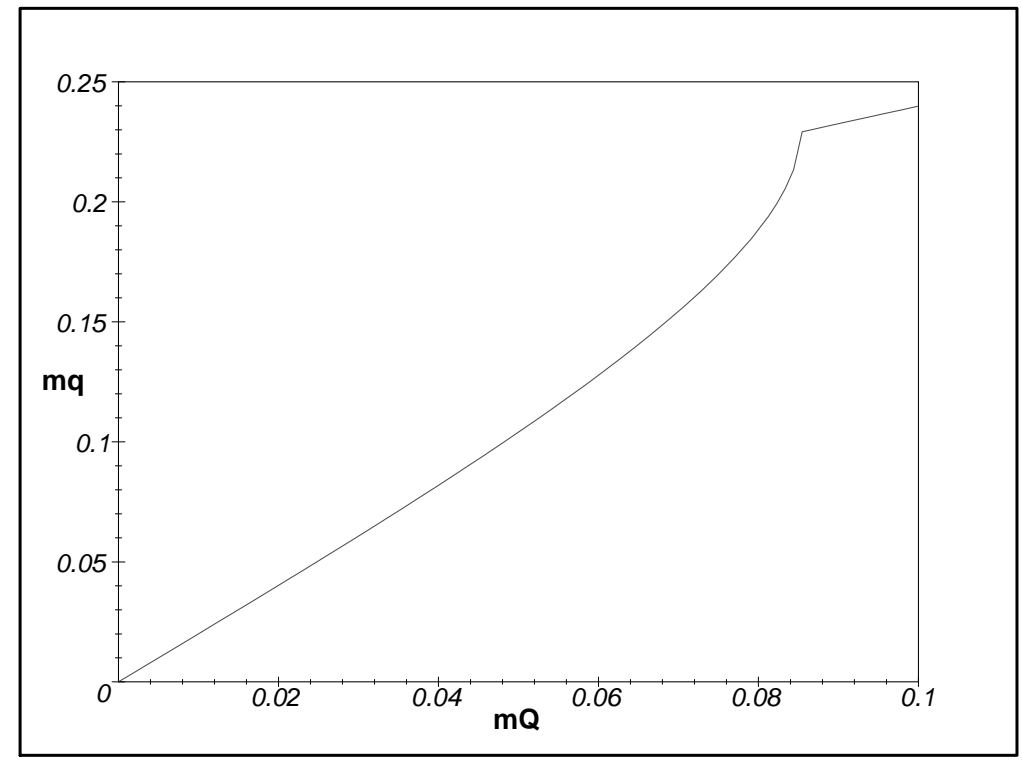

FIG. 5: A solution for the quark mass as a function of the Lagrangian mass, that satisfies $m_{q_{s}} \rightarrow 0$ when $m_{Q_{s}} \rightarrow 0$ (masses in units of $G e V / c^{2}$ ).

\section{TACHYONIC GLUON SELF-ENERGY}

Let us consider now the evaluation of the gluon self-energy. As it is known the exact gluon propagator can be written in the form

$$
G_{G}^{a b}(p)=\frac{\delta^{a b}}{p^{2}}\left(\frac{g_{\mu_{a} \mu_{b}}-\frac{p_{\mu_{a}} p_{\mu_{b}}}{p^{2}}}{1+\Pi\left(p^{2}\right)}+\alpha \frac{p_{\mu_{a}} p_{\mu_{b}}}{p^{2}}\right),
$$


where the function $\Pi$ is defined at the one loop level, by the expression

$$
\begin{aligned}
\Pi^{a b}\left(p^{2}\right) & =\Pi_{G}^{a b}+\Pi_{T}^{a b}+\Pi_{G h}^{a b}+\Pi_{Q}^{a b} \\
& =\delta^{a b}\left(p_{\mu_{a}} p_{\mu_{b}}-g_{\mu_{a} \mu_{b}} p^{2}\right) \Pi\left(p^{2}\right),
\end{aligned}
$$

which is diagrammatically represented in Figure 6 .

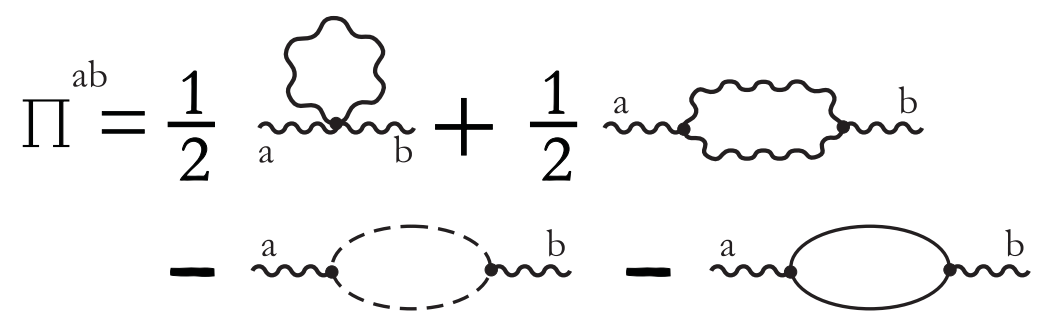

FIG. 6: Diagrams for $\Pi^{a b}\left(p^{2}\right)$.

Again, expanding in powers of the condensate parameter $C$, the total contribution can be decomposed in the standard one (associated to the ghost and the quark loop integrals) and the $C$ dependent ones $\Pi_{G}^{a b}$ and $\Pi_{T}^{a b}$ which can be readily calculated to be

$$
\begin{aligned}
\Pi_{G}^{a b} & =\frac{g^{2} C N}{(2 \pi)^{4}} \delta^{a b}\left(-5 g_{\mu_{a} \mu_{b}}+2 \frac{p_{\mu_{a}} p_{\mu_{b}}}{p^{2}}\right), \\
\Pi_{T}^{a b} & =\frac{3 g^{2} C N}{(2 \pi)^{4}} \delta^{a b} g_{\mu_{a} \mu_{b}} .
\end{aligned}
$$

Summing up these particular two terms leads up to

$$
\Pi_{C}^{a b}\left(p^{2}\right)=\delta^{a b}\left(p_{\mu_{a}} p_{\mu_{b}}-g_{\mu_{a} \mu_{b}} p^{2}\right) \frac{2 g^{2} C N}{(2 \pi)^{4} p^{2}} .
$$

Considering again our case the $S U(3)$ group, that is $N=3$, and disregarding in a first consideration the standard one loop contribution, the function $\Pi$ reduces to

$$
\Pi\left(p^{2}\right)=\frac{6 g^{2} C}{(2 \pi)^{4}} \frac{1}{p^{2}},
$$

which in turns leads out to the following condition for the poles of (19)

$$
p^{2}-m_{G}^{2}=0
$$

where

$$
m_{G}^{2}=-\frac{6 g^{2} C}{(2 \pi)^{4}}=-0.25\left(G e V / c^{2}\right)^{2} .
$$


Therefore, as the parameter $C$ has been defined before as real and positive [14, 15], it follows that the transverse gluon mass correction becomes tachyonic. The ability of a tachyonic mass in producing improvements in models for the inter quark potential has been recently argued in the literature [26, 27]. The effect is linked with the introduction of a linearly rising term as a first correction to the Coulomb potential in the massless case [27]. Therefore, the tachyonic result arising here for the gluon mass appears to be of interest for a derivation, within the present framework, of former successful phenomenological bound state models for mesons [35, 36, 37]. We are grateful to J.P. Vary and J. Qiu for the suggestion of this possibility. It should be also underlined that the tachyonic character of the gluon self-energy arising here, is fully compatible with the more general result of Fukuda for the normal Green functions in QCD, when gluon condensation is present [38]. This circumstance then suggests, that the modified expansion under discussion could be a sort of perturbative representation of the Fukuda non-perturbative treatment of the gluon condensation in QCD. In a different manner, the approach of Celenza and Shakin, and other authors can be expected to be related with a different initial state also incorporating gluon condensation. It could be the point that, at the end, both pictures can appear to be equivalent at the level of exact results. This could be the case because the physical degrees of freedom are not the gluons and quarks ones, but their colorless composite combinations which physics is determined by the bound state dispersion relations.

Finally in this section it deserves to be stressed that if a modification of the ghost propagator is present, then a mass terms for the longitudinal propagator could also appear and the polarization operator (gluon self-energy) becomes non-transverse. This result means a violation of a Ward identity representing the gauge invariance by the presence of ghost (and fermion) condensates. Thus, it follows that the selection of the parameter of the initial state in [14, 15] was the appropriate one for the satisfaction of a gauge invariance condition at this stage. The physical meaning of the alternative procedures of constructing the initial state needs however for clarification.

\section{SUMMARY}

The following results can be enumerated:

1) A calculation of the mean value of $G^{2}$ up to order $g^{2}$ in gluodynamics, was performed. 
The result coincided with a previous one obtained in a simpler approximation [13, 14] and which signals that quartic gluon self-interaction is relevant in fixing a non-vanishing gluon condensation parameter already in the first order of the perturbation theory [19].

3) The one loop corrections for the quark masses under the influence of the condensate were evaluated. When only the terms reflecting the effect of the condensate are considered, the results for the constituent masses showed a branch which is real as a function of all the values of the quark current mass. Another solution which is real up to a critical value of the Lagrangian masses, and having a vanishing proper mass in the limit of small current masses is also obtained. The former branch predicts values of one third of the nucleon mass for the masses of the light quarks. The effective quark masses for all the quark flavors were evaluated as determined by their known current mass in the mentioned approximation.

4) After the inclusion of the rest of the terms completing the full one loop contribution to the quark self-energy, an interesting outcome arises. The $u$ and $d$ quarks, although showing high peak values of the propagator components near the former values of the squared proper masses, become confined. That is, their propagators do not show pole structures. On the other hand the $s, c, b$ and $t$ quarks acquire poles but now not at real values of $p^{2}$. Therefore, these free modes are damped. These properties suggest a possibility for the explanation of the disparity of stability properties in the nucleons and higher resonances. It would work as follows, the absence of propagation solutions for the $u$ and $d$ quarks could assure the unlimited life time of nucleons. Moreover, the damped oscillations present for the rest of the quarks can create decay modes for the bound states composed of them.

5) By mean of the obtained mass values, the ground state energies selected within each of the groups hadron resonance reported in [21], were estimated through the simple addition of the masses of their known quark constituents. It could be remarked that the obtained values are reasonable ones in comparison with the data, after taking into account the high errors in the experimentally determined Lagrangian masses. This fact supports the hypothesis about the main glue nature of the constituent mass values of the light quarks. The small contribution of the binding potential energy of such resonance to their total mass is also indicated and thus suggests the applicability of non relativistic approximations in their study.

6) The contribution of the condensate to the gluon self-energy of order $g^{2}$ was calculated. A tachyonic result for the gluon mass arises for the parameter values employed in the construction of the initial state defined in [14, 15]. This conclusion is in concordance with the 
analysis of Fukuda for gluon condensation [38]. As it was remarked before, there are recent arguments claiming the possibility of a tachyonic gluon mass [18, 26, 27] and its role in improving the quark antiquark potential in bound state models of mesons [27.

It seems useful to comment here some basic issues related with the modified expansion being considered:

a) The Feynman expansion depending on the condensate parameter and taken for the gauge parameter $\alpha=1$ corresponds with the Wick expansion around a physical state of the interaction free QCD. In this somewhat limited way the arbitrariness of the gauge parameter in the result for the gluon mass in [13] was solved in [14, 15]. However, in order to furnish a fully consistent picture including renormalization, a physically equivalent version of the expansion for arbitrary values of $\alpha$ should developed. This question will be addressed in future works.

b) In [14, 15] the condensation parameter $C$ was defined as a real and positive one. This result determined the tachyonic character for the gluon mass found here. Further, the sign of $C$ also fixes the real values of the order of the one third of the nucleon mass for the constituent quark masses also calculated in the present work. The opposite sign produces confined quark modes.

c) An aspect which is important to underline is that the selection of the parameters defining the initial state in [14, 15] was designed also in order to impose the absence of a modification for the free ghost propagator. However, as it followed from the present analysis, this property in turns, is related with the fact that a condensate modification of the ghost propagator can produce a longitudinal contribution to the self-energy. But, such a term should not exist for the transversality condition of the polarization operator (a Ward identity) to be obeyed. Thus, its appearance could break manifestly the gauge invariance. Therefore, the present work is also given foundation to the non-modified ghost propagator choice considered in 113, 14, 15.

At the present stage we identify some important questions to be addressed in the further extension of the work:

1) To explore the possibility of showing the exact gauge parameter independence of the physical results within the modified expansion.

2) To address the general proof of the renormalizability in the expansion.

3) To search for a derivation of existing successful bounded state models for the heavy 
quark mesons [35, 36, 37], as realized by the a ladder approximation for the Bethe-Salpeter equation within the proposed modified expansion. The presence of a tachyonic gluon propagator in the approach (which is argued to have the effect of introducing a linearly rising component in the inter-quark potential [27]) and the obtained constituent values for the light quark masses already support such a possibility.

4) Improve the study of the effective action as a functional of the condensate parameter done in [13] by also including the gauge field in order to search for a variant of the leading logarithm model useful for the investigation of field configurations associated to inter-quark strings, nucleon-nucleon potentials, etc.

5) It can be underlined that the considered expansion seems that could furnish a general way for the perturbative description of pair condensates in Many Body theory. An important example coming to the mind is the BCS state of the usual superconductivity. Pair states being more closely connected with QCD were discussed in [39, 40]. In addition, in [40] it was advanced before the idea of using diquark condensates as generating an alternative for the Higgs mechanism.

Finally, we would like to conjecture about a possibility suggested by the results of this work. It is related with the question about whether the mass spectrum of the whole three generations of fundamental fermions could be predicted by a slight generalization of the modified perturbation expansion under consideration. In this sense, the given arguments led us to the idea that (after the introduction of quark condensates along the same lines as it was done for gluon ones in [13, 14, 15]) the received perturbation expansion can have the chance to predict both, the Lagrangian mass and the constituent quark mass spectra of the three families of fundamental fermions. The fermion condensates as described in the proposed perturbative way, would have the role of producing the Lagrangian quark masses, through the chiral symmetry breaking. The gluonic condensates, in one hand, and as illustrated here, could be responsible of generating states of large constituent mass for the low mass quarks states. In another hand, it seems feasible that the higher order radiative corrections (including color interactions with the condensate as well as chiral symmetry corrections) could also determine the mass spectra for leptons and neutrinos. The smaller scale for the masses of these particles could be produced by the lack of lower order either color or electric-weak interaction terms in their self-energy. Therefore, the possibility that the Lagrangian mass spectrum of the three generations of the fundamental fermions could 
be predicted by a modified perturbation expansion of the sort being proposed is suggested. Work directed to investigate this possibility will be considered elsewhere. The modified expansion under study could be a useful technique of implementing this idea.

\section{Acknowledgments}

The authors wish to deeply acknowledge the helpful advice and comments of Profess. J. Pestieau, J. Alfaro, M. Lowe, C. Friedli, M. Hirsch and A. González. One of the authors (A. C. M.) also would like to express his gratitude to the Abdus Salam ICTP, and in particular to its Associate-Membership Program for the whole general support. The support of the International Institute for Theoretical and Applied (IITAP) (UNESCO and Iowa State University) and the Christopher Reynolds Foundation during the completion of this work at IITAP is also greatly acknowledged. The suggestion of the possibility of applying the procedure for describing bound state model for mesons is acknowledged from Dr. J. P. Vary and Dr. J. Qiu. Finally, we are grateful to K. Tornés for the help in the preparation of the manuscript.

[1] G. K. Savvidy, Phys. Lett. B71, 133 (1977).

[2] I. A. Batalin, S. G. Matinyan, and G.K. Savvidy, Sov. J. Nucl. Phys. 26, 214 (1977).

[3] S. G. Matinyan, and G. K. Savvidy, Sov. J. Nucl. Phys. 25, 118 (1977); Nucl. Phys. B134, 539 (1978).

[4] A. Cabo, O.K. Kalashnikov and A.E. Shabad, Nucl. Phys. B185, 473 (1981).

[5] E. U. Shuryak, The QCD Vacuum, Hadrons and the Superdense Matter, World Scientific, Singapore, 1988.

[6] T. Schäfer and E. V. Shuryak, Rev. Mod. Phys. 70, 323 (1998).

[7] H. Fritzsch, M. Gell-Mann, and H. Leutwyler, Phys. Lett. B47, 365 (1973).

[8] D. J. Gross and F. Wilczec, Phys. Rev. D8, 3497 (1973).

[9] S. Weinberg, Phys. Rev. Lett. 31, 494 (1973).

[10] S. Weinberg, The Problem of Mass, Trans. New York Acad. Sci. 38: 185-201, 1977; HUTP77/A057, (1977). 
[11] S. Weinberg, Phys. Rev. Lett. 64, 1181 (1990).

[12] S. Weinberg, Phys. Rev. Lett. 67, 3473 (1991).

[13] A. Cabo, S. Peñaranda and R. Martinez, Mod. Phys. Lett. A10, 2413 (1995).

[14] M. Rigol and A. Cabo, Phys. Rev. D62, 074018 (2000); hep-th/9909057 (1999).

[15] M. Rigol, Acerca de un Estado de Vacío Alternativo para la QCD Perturbativa, Graduate Dissertation Thesis, Instituto Superior de Ciencias y Tecnología Nucleares, La Habana, Cuba, 1999, hep-th/0109012 (2001).

[16] P. Hoyer, NORDITA - 96/63 P (1996), hep-ph/9610270 (1996).

[17] P. Hoyer, NORDITA - 97/44 P (1997), hep-ph/9709444 (1997).

[18] P. Hoyer and J. Rathsman, JHEP 05, 020 (2001).

[19] M. A. Shifman, A. I. Vainshtein and V. I. Zakharov, Nucl. Phys. B147, 385 (1979); B147, 448 (1979); B 147, 519 (1979).

[20] T. Kugo and I. Ojima, Prog. Theor. Phys. Suppl. 66, 1 (1979).

[21] C. Caso, et.al., Review of Particle Physics, The European Physical Journal C 3, 1 (1998).

[22] H. J. Munczek and A. M. Nemirovsky, Phys. Rev. D28, 181 (1983).

[23] C. J. Burden, C. D. Roberts and A. G. Williams, Phys. Lett. B285, 347 (1992).

[24] L. S. Celenza and C. M. Shakin, Phys. Rev. D34, 1591 (1986).

[25] H. P. Pavel, D. Blaschke, V. N. Pervushin and G. Röpke, Int. J. Mod. Phys. A14, 205 (1999).

[26] K. G. Chetyrkin, S. Narison and V. I. Zakharov, Nucl. Phys. B550, 353 (1999).

[27] S. J. Huber, M. Reuter and M. G. Schmidt, Phys. Lett. B462, 158 (1999).

[28] T. Muta, Foundations of Quantum Chromodynamics, World Scientific Lectures Notes in Physics - Vol. 5, Singapore, 1987.

[29] L. D. Faddeev and A. A. Slanov, Gauge Fields. Introduction to Quantum Theory, Benjamin Cummings Publishing, Reading, Mass., 1980.

[30] S. Randjbar-Daemi, Course in Quantum Field Theory, Lectures Notes ICTP Diploma Programme, ICTP, Trieste 1994.

[31] S. Narison,Phys. Rep. 84, 263 (1982).

[32] J. C. Collins, Renormalization, Cambridge University Press, Cambridge, 1987.

[33] V. Elias, T. G. Steele and M. Scadron, Phys. Rev. D38, 1584 (1988).

[34] V. Elias and T.G. Steele, Phys. Lett. B212, 88 (1988).

[35] A. J. Sommerer, J. R. Spence and J. P. Vary, Phys. Rev. C49, 513 (1994). 
[36] A. J. Sommerer, A. Abd El-Hady, J. R. Spence and J. P. Vary, Phys. Lett. B348, 277 (1995).

[37] A. Abd El-Hady, A. A. K. Lodhi and J. P. Vary, Phys. Rev. D59, 094001-1 (1999).

[38] R. Fukuda, Phys. Rev. D21, 485 (1980).

[39] A. Mishra, H. Mishra, V. Sheel, S. P. Misra and P. K. Panda, Int. J. Mod. Phys. E5, 93 (1996), hep-ph/9404255 (1994).

[40] D. Diakonov, Phys. Lett. B373, 147 (1996), hep-ph/9512385 (1995). 